\title{
Curvature and critical Riemannian metric
}

\author{
By Yosio MUTō
}

(Received June 18, 1973)

(Revised Oct. 28, 1973)

Let $M$ be a compact orientable $C^{\infty}$ manifold and $g$ a $C^{\infty}$ Riemannian metric on $M$ satisfying

$$
\int_{M} d V=1
$$

where $d V$ is the volume element of $M$ measured by $g$. We denote the set of all such metrics by $\mathscr{M}(M)$ or $\mathscr{M}$. When $g$ is fixed we have a Riemannian manifold $(M, g)$.

Let us take a covering $\{U\}$ of $M$ by coordinate neighborhoods and denote the local coordinates in $U$ by $x^{h}$. In each neighborhood $U$ we use the natural frame. Then the components of the curvature tensor of $(M, g)$ in $U$ is given by

$$
K_{k j i}{ }^{h}=\partial_{k}\left\{\begin{array}{l}
h \\
j i
\end{array}\right\}-\partial_{j}\left\{\begin{array}{l}
h \\
k i
\end{array}\right\}+\left\{\begin{array}{c}
h \\
k p
\end{array}\right\}\left\{\begin{array}{c}
p \\
j i
\end{array}\right\}-\left\{\begin{array}{l}
h \\
j p
\end{array}\right\}\left\{\begin{array}{c}
p \\
k i
\end{array}\right\}
$$

where $\left\{\begin{array}{c}h \\ j i\end{array}\right\}$ are the Christoffel symbols derived from the components $g_{j i}$ of $g$, Latin indices run the range $\{1, \cdots, n\}$, and the summation convention is adopted. The Ricci tensor and the scalar curvature are given respectively by

$$
K_{j i}=K_{p j i}^{p}, \quad K=g^{j i} K_{j i}
$$

where $g^{j i}$ are defined by $g_{i k} g^{k h}=\delta_{i}^{n}$. Similarly all tensors will be expressed in terms of their components.

In a Riemannian manifold $(M, g)$ indices can be raised and lowered by $g^{j i}$ and $g_{j i}$ so that for example $K^{k j i h}=K_{d c b}{ }^{h} g^{d k} g^{c j} g^{b i}$ are the contravariant components of the curvature tensor. Thus $K_{k j i n} K^{k j i n}$ is a scalar. Considering this at each point of $M$ we get a scalar field.

Now let us consider the integral

$$
J[g]=\int_{M} K_{g} d V_{g},
$$

where we write $K_{g}$ and $d V_{g}$ for $K$ and $d V$ respectively in order to emphasize that these depend on the metric $g$. $J[g]$ is the image of $g$ by a map $J: \mathscr{M}(M) \rightarrow \boldsymbol{R}$. Critical points of this map are known as Einstein metrics. 
M. Berger studied the second derivative of $J[g(t)]$ for curves $g(t)$ of $\mathscr{M}(M)$ and showed that it is not true that the index of $J[g]$ and also the index of $-J[g]$ are finite for every critical point [1]. Recently the present author proved that the index of $J[g]$ and the index of $-J[g]$ are both positive at each critical point $[6]$. This result diminishes our interest in $J$ to a certain extent.

Let us consider the integral

$$
I[g]=\int_{M} K_{k j i h} K^{k j i h} d V_{g}
$$

Then $I$ is also a mapping $I: \mathscr{M}(M) \rightarrow \boldsymbol{R}$. This integral has a remarkable property that $I[g]$ is non-negative and moreover that, if $M$ does not admit a locally flat metric, then $I[g]$ is strictly positive.

If $\eta$ is a diffeomorphism of $M$ and $\eta^{*}$ its pull back, we have $I[g]=$ $I\left[\eta^{*}(g)\right]$. Hence we can deduce a mapping $\tilde{I}: \mathscr{M} / \mathscr{D} \rightarrow \boldsymbol{R}$ from the mapping $I: \mathscr{M} \rightarrow \boldsymbol{R}$ where $\mathscr{D}$ is the diffeomorphism group of $M$ and $\mathscr{M} / \mathscr{D}$ is the space of orbits generated by $\mathscr{D}$ of Riemannian metrics [2].

If $\bar{g}$ is a critical point of $I$, then the orbit of $\bar{g}$ by $\mathscr{D}$ is a critical point of $\tilde{I}$ and vice versa. In this case let us say that $\bar{g}$ is a critical point of $\tilde{I}$ for convenience sake. This convention is useful since there can exist no local minimum, in the strict sense, of the mapping $I$ but $\tilde{I}$ may possibly have a local minimum and the present paper concerns this.

Some years ago M. Berger obtained differential equations of $g$ for the critical point, namely the critical Riemannian metric, of the mapping $I[1]$. In the present paper it is shown that a metric of constant curvature is a critical Riemannian metric. Moreover, the second derivative of $I[g(t)]$ at a critical point is calculated and the following Main Theorem is obtained.

THEOREM. If $M$ is diffeomorphic to $S^{n}$ and $\bar{g}$ is a metric of positive constant curvature on $M$, then the index of $I$ and also of $\tilde{I}$ at $\bar{g}$ is zero and $\widetilde{I}$ has a local minimum at $\bar{g}$.

\section{$\S 1$. Critical Riemannian metric.}

Here and in the sequel we write

$$
I[g]=\int K_{k j i n} K^{k j i h} d V .
$$

We have dropped $M$ and $g$ in this formula. A $C^{\infty}$ curve in $\mathcal{M}$ will be represented locally by $g_{j i}\left(x^{1}, \cdots, x^{n} ; t\right)$ and we define a tensor field $D_{j i}$ on $(M, g(t))$ by

$$
D_{j i}(x, t)=\frac{\partial g_{j i}(x, t)}{\partial t}
$$


This symmetric $(0,2)$-tensor satisfies

$$
\int D_{p}^{p} d V=0
$$

because of $(0.1)$.

The curvature tensor $K_{k j i}{ }^{h}$ changes with $g$ and we get [6]

$$
\frac{\partial}{\partial t} K_{k j i}{ }^{h}=\nabla_{k} D_{j i}{ }^{h}-\nabla_{j} D_{k i}{ }^{h}
$$

where the tensor $D_{j i}{ }^{h}$ is defined by

and satisfies

$$
D_{j i}^{h}=\frac{\partial}{\partial t}\left\{\begin{array}{l}
h \\
j i
\end{array}\right\}
$$

$$
D_{j i}{ }^{h}=\frac{1}{2}\left(\nabla_{j} D_{i}{ }^{h}+\nabla_{i} D_{j}{ }^{h}-\nabla^{h} D_{j i}\right)
$$

and $\nabla$ means covariant differentiation with respect to the metric tensor $g(t)$. As we have

$$
\frac{\partial}{\partial t} g^{i n}=-g^{k i} g^{j h} \frac{\partial}{\partial t} g_{k j}=-D^{i n},
$$

we get

$$
\begin{aligned}
\frac{\partial}{\partial t}\left(K_{k j i h} K^{k j i h}\right)= & \frac{\partial}{\partial t}\left(K_{k j i}{ }^{h} K_{d c b}{ }^{a}{ }^{k d} g^{j c} g^{i b} g_{h a}\right) \\
= & 2\left(\nabla_{k} D_{j i}{ }^{h}-\nabla_{j} D_{k i}{ }^{h}\right) K^{k j i}{ }_{h} \\
& +K_{k j i}{ }^{h} K_{d c b}{ }^{a}\left(-D^{k d} g^{j c} g^{i b} g_{h a}-g^{k d} D^{j c} g^{i b} g_{h a}\right. \\
& \left.\quad-g^{k d} g^{j c} D^{i b} g_{h a}+g^{k d} g^{j c} g^{i b} D_{h a}\right) .
\end{aligned}
$$

Substituting (1.5) into this formula and taking some property of the curvature tensor into account, we can deduce

$$
\frac{\partial}{\partial t}\left(K_{k j i h} K^{k j i h}\right)=4 K_{h}^{k j i} \nabla_{k} \nabla_{i} D_{j}{ }^{h}-2 K_{k j i}{ }^{b} K^{k j i a} D_{b a} .
$$

Now, from (1.1) we get

$$
\frac{d}{d t} I[g(t)]=\int\left[\frac{\partial}{\partial t}\left(K_{k j i n} K^{k j i n}\right)+\frac{1}{2} K_{k j i h} K^{k j i n} g^{q p} D_{q p}\right] d V .
$$

Substituting (1.6) into this and applying Green's theorem, we get

$$
\begin{aligned}
\frac{d}{d t} I[g(t)]=\int[ & 4\left(\nabla_{i} \nabla_{k} K^{k j i}{ }_{h}\right) D_{j}{ }^{h} \\
& \left.\quad-2 K_{k j i}{ }^{q} K^{k j i p} D_{q p}+\frac{1}{2} K_{k j i n} K^{k j i n} D_{p}{ }^{p}\right] d V .
\end{aligned}
$$

Then, applying Ricci's identity and Bianchi's identity, we get 


$$
\begin{aligned}
\frac{d}{d t} I[g(t)]=\int\left[2 \nabla^{j} \nabla^{i} K-4 \nabla_{p} \nabla^{p} K^{j i}+4 K_{p}^{j} K^{p i}\right. \\
\\
\left.-4 K_{q p}^{j}{ }^{i} K^{q p}-2 K^{s r q j} K_{s r q}{ }^{i}+\frac{1}{2} K_{d c b a} K^{d c b a} g^{j i}\right] D_{j i} d V .
\end{aligned}
$$

A point $\bar{g}$ is a critical point of $I$ if and only if (1.7) vanishes at $t=0$ for all curves $g(t)$ such that $g(0)=\bar{g}$. Let us consider the integral which is obtained from the right-hand member of (1.7) by replacing $g(t)$ with $\bar{g}$. Then we can say that $\bar{g}$ is a critical point of $I$ if and only if this integral vanishes for all tensor fields $D_{j i}$ satisfying (1.3) in which $g$ is replaced with $\bar{g}$. Thus we see that $\bar{g}$ is a critical point of $I$ if and only if

$$
\begin{aligned}
2 \nabla^{j} \nabla^{i} K-4 \nabla_{p} \nabla^{p} K^{j i} & +4 K^{j}{ }_{p} K^{p i}-4 K^{j}{ }_{q p}{ }^{i} K^{q p} \\
& -2 K^{s r q j} K_{s r q}{ }^{i}+\frac{1}{2}-K_{d c b a} K^{d c b a} g^{j i}=c g^{j i}
\end{aligned}
$$

is satisfied by $g=\bar{g}$ and some constant $c$. Precisely, $c$ is obtained by transvecting with $g_{j i}$, hence

$$
c=-\frac{2}{n} \nabla_{p} \nabla^{p} K+\left(\frac{1}{2}-\frac{2}{n}\right) K_{d c b a} K^{d c b a} .
$$

Thus we get the following theorem [1].

THEOREM 1.1. Let $M$ be a compact orientable $C^{\infty}$ manifold and $\mathscr{M}$ be the space of Riemannian metrics on $M$ satisfying (0.1). Then a necessary and sufficient condition for a Riemannian metric $g$ to be a critical point of the functional (1.1) is that (1.8) is satisfied by this metric $g$ where $c$ is a constant in $M$. At that time $c$ is given by (1.9).

ExAmple. If $M$ is diffeomorphic to $S^{n}, M$ admits a Riemannian metric of positive constant curvature. Then

$$
K_{k j i h}=\frac{K}{n(n-1)}\left(g_{k h} g_{j i}-g_{j n} g_{k i}\right)
$$

satisfies (1.8) with

$$
c=\left(\frac{1}{2}-\frac{2}{n}\right) \frac{2 K^{2}}{n(n-1)} .
$$

Hence this metric is a critical point of the functional (1.1) with the critical value

$$
\frac{2 K^{2}}{n(n-1)}
$$

Now, the purpose of the present paper is to prove the following theorem which is equivalent with the Main Theorem stated in the introduction.

THEOREM 1.2. Let $M$ be a $C^{\infty}$ manifold diffeomorphic to $S^{n}$. Then the mapping $\tilde{I}: \mathscr{M} / \mathscr{D} \rightarrow \boldsymbol{R}$ given by the functional (1.1) has a local minimum at the Riemannian metric $\bar{g}$ of positive constant curvature (1.10). 


\section{$\S 2$. The second derivative of $I[g(t)]$.}

In order to prove the main theorem let us first calculate the second derivative $I^{\prime \prime}[g(t)]$ for an arbitrary curve $g(t)$ of $\mathscr{M}$.

If we define $W^{j i}$ by

$$
\begin{aligned}
W^{j i}= & 2 \nabla^{j} \nabla^{i} K-4 \nabla_{p} \nabla^{p} K^{j i}+4 K^{j}{ }_{p} K^{p i}-4 K^{j}{ }_{q p}{ }^{i} K^{q p} \\
& -2 K^{s r q j} K_{s r q}{ }^{i}+\frac{1}{2} K_{d c b a} K^{d c b a} g^{j i},
\end{aligned}
$$

we get

$$
I^{\prime \prime}[g(t)]=\int\left[\frac{\partial W^{j i}}{\partial t} \frac{\partial g_{j i}}{\partial t}+W^{j i}\left(\frac{\partial^{2} g_{j i}}{\partial t^{2}}+\frac{1}{2} \frac{\partial g_{j i}}{\partial t} g^{q p} \frac{\partial g_{q p}}{\partial t}\right)\right] d V
$$

where

$$
\begin{aligned}
\frac{\partial W^{j i}}{\partial t}= & 2 \frac{\partial}{\partial t} \nabla^{j} \nabla^{i} K-4 \frac{\partial}{\partial t} \nabla_{p} \nabla^{p} K^{j i} \\
& +4 \frac{\partial}{\partial t}\left(K_{p}^{j} K^{p i}\right)-4 \frac{\partial}{\partial t}\left(K_{q p}^{j} K^{i p}\right)-2 \frac{\partial}{\partial t}\left(K^{s r q j} K_{s r q}{ }^{i}\right) \\
& +\frac{1}{2} \frac{\partial}{\partial t}\left(K_{d c b a} K^{d c b a} g^{j i}\right) .
\end{aligned}
$$

From the definition of $D_{j i}{ }^{h}$ we have the following identity which is valid for any $C^{\infty}$ tensor field depending on $t$ differentiably, for example $T_{i}{ }^{h}$,

$$
\frac{\partial}{\partial t} \nabla_{j} T_{i}{ }^{h}=\nabla_{j} \frac{\partial}{\partial t} T_{i}{ }^{h}-D_{j i}{ }^{p} T_{p}{ }^{h}+D_{j p}{ }^{h} T_{i}{ }^{p} .
$$

Applying this identity to the first and the second terms in the right-hand member of (2.3), we get

$$
\begin{aligned}
\frac{\partial}{\partial t} \nabla^{j} \nabla^{i} K= & \frac{\partial}{\partial t}\left(g^{j q} g^{i p} \nabla_{q} \nabla_{p} K\right) \\
= & -D^{j p} \nabla_{p} \nabla^{i} K-D^{i p} \nabla^{j} \nabla_{p} K \\
& +\nabla^{j} \nabla^{i} \frac{\partial}{\partial t} K-D^{j i p} \nabla_{p} K, \\
\frac{\partial}{\partial t} \nabla_{p} \nabla^{p} K^{j i}= & \frac{\partial}{\partial t}\left(g^{q p} \nabla_{q} \nabla_{p} K^{j i}\right) \\
= & -D^{q p} \nabla_{q} \nabla_{p} K^{j i} \\
& +g^{q p}\left(-D_{q p} \nabla_{r} K^{j i}+D_{q r}^{j} \nabla_{p} K^{r i}+D_{q r}{ }^{i} \nabla_{p} K^{j r}\right) \\
& +g^{q p} \nabla_{q}\left(\nabla_{p} \frac{\partial}{\partial t} K^{j i}+D_{p r}^{j} K^{r i}+D_{p r}^{i} K^{j r}\right) .
\end{aligned}
$$

From (1.4) and (1.5) we get 


$$
\begin{aligned}
\frac{\partial}{\partial t} K^{j i}= & -D^{j p} K_{p}{ }^{i}-D^{i p} K_{p}^{j} \\
& +\frac{1}{2}\left(\nabla_{p} \nabla^{j} D^{i p}+\nabla_{p} \nabla^{i} D^{j p}-\nabla_{p} \nabla^{p} D^{j i}-\nabla^{j} \nabla^{i} D_{p}^{p}\right), \\
\frac{\partial}{\partial t} K=- & D^{q p} K_{q p}+\nabla_{q} \nabla_{p} D^{q p}-\nabla_{q} \nabla^{q} D_{p}^{p}, \\
\frac{\partial}{\partial t}\left(K^{j}{ }_{p} K^{p i}\right) & \\
= & -D^{j q} K_{q p} K^{p i}-D^{i q} K_{q p} K^{p j}-K^{j q} D_{q p} K^{p i} \\
& +\frac{1}{2} K^{q i}\left(\nabla_{p} \nabla^{j} D_{q}{ }^{p}+\nabla_{p} \nabla_{q} D^{j p}-\nabla_{p} \nabla^{p} D_{q}^{j}-\nabla_{q} \nabla^{j} D_{p}^{p}\right) \\
& +\frac{1}{2} K^{q j}\left(\nabla_{p} \nabla^{i} D_{q}{ }^{p}+\nabla_{p} \nabla_{q} D^{i p}-\nabla_{p} \nabla^{p} D_{q}{ }^{i}-\nabla_{q} \nabla^{i} D_{p}^{p}\right),
\end{aligned}
$$

$$
\begin{aligned}
& \frac{\partial}{\partial t}\left(K^{j}{ }_{q p}{ }^{i} K^{q p}\right) \\
& =-K_{r q p}{ }^{i} K^{q p} D^{r j}-K_{q p}^{j}{ }^{i}\left(D^{q r} K_{r}{ }^{p}+D^{p r} K_{r}{ }^{q}\right) \\
& \quad+\frac{1}{2} K^{q p}\left(\nabla^{j} \nabla_{q} D_{p}{ }^{i}+\nabla^{j} \nabla_{p} D_{q}{ }^{i}-\nabla^{j} \nabla^{i} D_{q p}\right. \\
& \left.\quad-\nabla_{q} \nabla^{j} D_{p}{ }^{i}-\nabla_{q} \nabla_{p} D^{j i}+\nabla_{q} \nabla^{i} D_{p}^{j}\right) \\
& \quad+\frac{1}{2} K_{q p}^{j}\left(\nabla_{r} \nabla^{q} D^{p r}+\nabla_{r} \nabla^{p} D^{q r}-\nabla_{r} \nabla^{r} D^{q p}-\nabla^{q} \nabla^{p} D_{r}^{r}\right),
\end{aligned}
$$

$$
\frac{\partial}{\partial t}\left(K^{s r q j} K_{s r q}^{i}\right)
$$

$$
\begin{aligned}
= & \left(\nabla_{c} \nabla_{b} D_{a}^{j}+\nabla_{c} \nabla_{a} D_{b}^{j}-\nabla_{c} \nabla^{j} D_{b a}\right) K^{c b a i} \\
& +\left(\nabla_{c} \nabla_{b} D_{a}{ }^{i}+\nabla_{c} \nabla_{a} D_{b}{ }^{i}-\nabla_{c} \nabla^{i} D_{b a}\right) K^{c b a j} \\
& -K^{b s r j} K^{a}{ }^{i}{ }^{i} D_{b a}-K^{s b r j} K_{s}{ }^{a}{ }^{i} D_{b a} \\
& -K^{s r b j} K_{s r}{ }^{a i} D_{b a},
\end{aligned}
$$

$$
\begin{aligned}
\frac{\partial}{\partial t}( & \left.K_{d c b a} K^{d c b a} g^{j i}\right) \\
= & \left(4 K^{d c b a} \nabla_{d} \nabla_{b} D_{c a}-2 K_{d c b}{ }^{q} K^{d c b p} D_{q p}\right) g^{j i} \\
& -K_{d c b a} K^{d c b a} D^{j i} .
\end{aligned}
$$

Applying (1.5), (2.7) and (2.8) we get from (2.5) and $(2.6)^{1)}$

$$
\begin{aligned}
& \frac{\partial}{\partial t} \nabla^{j} \nabla^{i} K \\
&=-D^{j q} \nabla_{q} \nabla^{i} K-D^{i q} \nabla_{q} \nabla^{j} K \\
&+\nabla^{j} \nabla^{i}\left(-D^{q p} K_{q p}+\nabla_{q} \nabla_{p} D^{q p}-\nabla_{q} \nabla^{q} D_{p}^{p}\right) \\
&-\frac{1}{2}\left(\nabla^{j} D^{i q}+\nabla^{i} D^{j q}-\nabla^{q} D^{j i}\right) \nabla_{q} K,
\end{aligned}
$$

1) In this paper $\nabla A B$ always means $(\nabla A) B$. Thus we have $\nabla(A B)=\nabla A B+A \nabla B$. 


$$
\begin{aligned}
& \frac{\partial}{\partial t} \nabla_{q} \nabla^{q} K^{j i} \\
&=-D^{q p} \nabla_{q} \nabla_{p} K^{j i}-\nabla_{q} D^{q r} \nabla_{r} K^{j i}+\frac{1}{2} \nabla^{r} D_{q}^{q} \nabla_{r} K^{j i} \\
&+\left(\nabla_{q} D_{r}{ }^{j}+\nabla_{r} D_{q}{ }^{j}-\nabla^{j} D_{q r}\right) \nabla^{q} K^{r i} \\
&+\left(\nabla_{q} D_{r}{ }^{i}+\nabla_{r} D_{q}{ }^{i}-\nabla^{i} D_{q r}\right) \nabla^{q} K^{r j} \\
&+\frac{1}{2} \nabla^{p}\left(\nabla_{p} D_{r}{ }^{j}+\nabla_{r} D_{p}{ }^{j}-\nabla^{j} D_{p r}\right) K^{r i} \\
&+\frac{1}{2} \nabla^{p}\left(\nabla_{p} D_{r}{ }^{i}+\nabla_{r} D_{p}{ }^{i}-\nabla^{i} D_{p r}\right) K^{r j} \\
&-\nabla_{q} \nabla^{q}\left(D^{j s} K_{s}^{i}+D^{i s} K_{s}{ }^{j}-\frac{1}{2} \nabla_{s} \nabla^{j} D^{i s}-\frac{1}{2} \nabla_{s} \nabla^{i} D^{j s}\right. \\
&\left.\quad+\frac{1}{2} \nabla_{s} \nabla^{s} D^{j i}+\frac{1}{2} \nabla^{j} \nabla^{i} D_{s}^{s}\right) .
\end{aligned}
$$

Let us define $F$ by

$$
F=\frac{\partial W^{j i}}{\partial t} D_{j i}
$$

and the notation $\cong$ by $A \cong A+$ divergence. Then we get

$$
\begin{aligned}
& F \cong D_{j i}\left[-4 D^{j q} \nabla_{q} \nabla^{i} K\right. \\
& +2 \nabla^{j} \nabla^{i}\left(-D^{q p} K_{q p}+\nabla_{q} \nabla_{p} D^{q p}-\nabla_{q} \nabla^{q} D_{p}^{p}\right) \\
& -2 \nabla^{j} D^{i q} \nabla_{q} K+\nabla^{q} D^{j i} \nabla_{q} K+4 D^{q p} \nabla_{q} \nabla_{p} K^{j i} \\
& +4 \nabla_{q} D^{q p} \nabla_{p} K^{j i}-2 \nabla^{q} D_{p}^{p} \nabla_{q} K^{j i} \\
& -8\left(\nabla_{q} D_{p}^{j}+\nabla_{p} D_{q}^{j}-\nabla^{j} D_{q p}\right) \nabla^{q} K^{p i} \\
& -4 \nabla^{q}\left(\nabla_{q} D_{p}^{j}+\nabla_{p} D_{q}^{j}-\nabla^{j} D_{q p}\right) K^{p i} \\
& +2 \nabla_{q} \nabla^{q}\left(4 D^{j p} K_{p}{ }^{i}-2 \nabla_{p} \nabla^{j} D^{i p}+\nabla_{p} \nabla^{p} D^{j i}+\nabla^{j} \nabla^{i} D_{p}{ }^{p}\right) \\
& -8 D^{j q} K_{q p} K^{p i}-4 K^{j q} D_{q p} K^{p i} \\
& +4 K^{j q}\left(\nabla_{p} \nabla^{i} D_{q}^{p}+\nabla_{p} \nabla_{q} D^{i p}-\nabla_{p} \nabla^{p} D_{q}{ }^{i}-\nabla_{q} \nabla^{i} D_{p}^{p}\right) \\
& +4 K_{r q p}{ }^{i} K^{q p} D^{r j}+4 K_{q p}^{j}{ }^{i}\left(D^{q r} K_{r}^{p}+D^{p r} K_{r}^{q}\right) \\
& -2 K^{q p}\left(\nabla^{j} \nabla_{q} D_{p}{ }^{i}+\nabla^{j} \nabla_{p} D_{q}{ }^{i}-\nabla^{j} \nabla^{i} D_{q p}\right. \\
& \left.-\nabla_{q} \nabla^{j} D_{p}{ }^{i}-\nabla_{q} \nabla_{p} D^{j i}+\nabla_{q} \nabla^{i} D_{p}^{j}\right) \\
& -2 K^{j}{ }_{q p}{ }^{i}\left(\nabla_{r} \nabla^{q} D^{p r}+\nabla_{r} \nabla^{p} D^{q r}-\nabla_{r} \nabla^{r} D^{q p}-\nabla^{q} \nabla^{p} D_{r}^{r}\right) \\
& -4 K^{c b a j}\left(\nabla_{c} \nabla_{b} D_{a}{ }^{i}+\nabla_{c} \nabla_{a} D_{l}{ }^{i}-\nabla_{c} \nabla^{i} D_{b a}\right) \\
& +4 K^{b q p j} K^{a}{ }_{q p}^{i} D_{b a}+2 K^{q p b j} K_{q p}{ }^{a i} D_{b a} \\
& +\left(2 K^{d c b a} \nabla_{d} \nabla_{b} D_{c a}-K_{d c b}{ }^{q} K^{d c b p} D_{q p}\right) g^{j i} \\
& \left.-\frac{1}{2} K_{d c b a} K^{d c b a} D^{j i}\right] \text {. }
\end{aligned}
$$


This formula is valid for any value of $t$.

We now want to evaluate the integral (2.2) at $t=0$, namely, at a critical point.

As we have $W^{j i}=c g^{j i}$ at $t=0$, we get

$$
I^{\prime \prime}[g(0)]=\int\left[F+c g^{j i}\left(\frac{\partial^{2} g_{j i}}{\partial t^{2}}+\frac{1}{2} \frac{\partial g_{j i}}{\partial t} g^{q p} \frac{\partial g_{q p}}{\partial t}\right)\right]_{0} d V
$$

where []$_{0}$ means that we have put $t=0$.

From (0.1) we get

$$
\int\left[g^{j i} \frac{\partial^{2} \mathrm{~g}_{j i}}{\partial t^{2}}-D^{j i} D_{j i}+\frac{1}{2}\left(D_{p}^{p}\right)^{2}\right] d V=0 .
$$

Hence we can deduce

$$
I^{\prime \prime}[g(0)]=\int G d V
$$

where $G$ is given by

$$
G \cong\left[F+\left\{-\frac{2}{n} \nabla_{q} \nabla^{a} K+\left(\frac{1}{2}-\frac{2}{n}\right) K_{d c b a} K^{d c b a}\right\} D_{j i} D^{j i}\right]_{0},
$$

for we have (1.9).

\section{$\S 3$. The integrand of $I^{\prime \prime}[g(0)]$.}

According to M. Berger and D. Ebin [1], [2] we can deduce properties of the Hessian of $I[g]$ at a critical point $\bar{g}$ if we study $I^{\prime \prime}[g(0)]$ for all curves $g(t)$ such that $g(0)=\bar{g}$ and such that the tensor field $D_{j i}=\left[\partial g_{j i} / \partial t\right]_{0}$. satisfies $\nabla^{j} D_{j i}=0$.

If $D_{j i}$ satisfies

$$
\nabla^{j} D_{j i}=0
$$

we get

$$
\nabla_{q} \nabla^{j} D^{q i}=K^{j}{ }_{q} D^{q i}+K_{q}^{j}{ }_{p}^{i} D^{q p}
$$

and $D_{j i} \nabla^{j} T^{i} \cong 0$ for any vector field $T^{h}$ on $M$. Applying such results we get after some straightforward calculation

$$
\begin{aligned}
G \cong & -4 D_{j i} D^{j q} \nabla_{q} \nabla^{i} K-2 D_{j i} \nabla^{j} D^{i q} \nabla_{q} K \\
& +D_{j i} \nabla^{q} D^{j i} \nabla_{q} K+4 D_{j i} D^{q p} \nabla_{q} \nabla_{p} K^{j i} \\
& -2 \nabla^{q} D_{p}^{p} D_{j i} \nabla_{q} K^{j i} \\
& -8 D_{j i}\left(\nabla_{q} D_{p}^{j}+\nabla_{p} D_{q}^{j}-\nabla^{j} D_{q p}\right) \nabla^{q} K^{p i} \\
& -4 D_{j i}\left(\nabla^{q} \nabla_{q} D_{p}^{j}+K_{p}^{q} D_{q}^{j}-K^{j q} D_{q p}\right) K^{p i}
\end{aligned}
$$




$$
\begin{aligned}
& +2 \nabla_{q} \nabla^{q} D_{j i}\left(4 D^{j p} K_{p}{ }^{i}-2 K_{r}^{j}{ }_{p}^{i} D^{r p}-2 K_{p}^{j} D^{i p}+\nabla^{j} \nabla^{i} D_{p}^{p}+\nabla_{p} \nabla^{p} D^{j \imath}\right) \\
& -8 D_{j i} D^{j q} K_{q p} K^{p i} \\
& +4 D_{j i} K^{j q}\left(K_{q p} D^{i p}-\nabla_{p} \nabla^{p} D_{q}{ }^{i}-\nabla_{q} \nabla^{i} D_{p}{ }^{p}\right) \\
& +4 K_{r q p}{ }^{i} K^{q p} D^{r j} D_{j i} \\
& -2 K^{q p}\left(2 D_{j i} \nabla^{j} \nabla_{q} D_{p}{ }^{i}-D_{j i} \nabla^{j} \nabla^{i} D_{q p}-D_{j i} \nabla_{q} \nabla_{p} D^{j i}\right) \\
& -2 D_{j i} K^{j}{ }_{q p}{ }^{i}\left\{2\left(K_{r}{ }^{q}{ }^{p} D^{a r}+K_{r}^{q} D^{p r}\right)-\nabla_{r} \nabla^{r} D^{q p}-\nabla^{q} \nabla^{p} D_{r}{ }^{r}\right\} \\
& +2 D_{j i} K^{c b a j} K_{c b a} D_{p}{ }^{i} \\
& -4 D_{j i} K^{c b a j}\left(\nabla_{c} \nabla_{a} D_{b}{ }^{i}-\nabla_{c} \nabla^{i} D_{b a}\right) \\
& +4 K^{b q p j} K^{a}{ }_{q p}{ }^{i} D_{b a} D_{j i} \\
& +2 K^{d c b a} \nabla_{d} \nabla_{b} D_{c a} D_{p}{ }^{p} \\
& -K_{d c b}{ }^{q} K^{d c b p} D_{q p} D_{r}^{r} \\
& +\left(-\frac{2}{n} \nabla_{p} \nabla^{p} K-\frac{2}{n} K_{d c b a} K^{d c b a}\right) D_{j i} D^{j i}
\end{aligned}
$$

at $t=0$, that is, at $\bar{g}=g(0)$.

$\S 4$. $I^{\prime \prime}[g(0)]$ when $g(0)$ is the metric of constant curvature.

If $g(0)$ is a metric of constant curvature, we have

$$
\begin{aligned}
K_{k j i h} & =\frac{K}{n(n-1)}\left(g_{k h} g_{j i}-g_{j h} g_{k i}\right), \\
K^{d c b j} K_{d c b}{ }^{i} & =\frac{2 K^{2}}{n^{2}(n-1)} g^{j i}, \\
K^{k q p j} K_{q p}^{i}{ }^{h} & =\frac{K^{2}}{n^{2}(n-1)^{2}}\left\{(n-2) g^{k j} g^{i n}+g^{k i} g^{j h}\right\}
\end{aligned}
$$

and $\nabla_{k} K^{j i}=0, \nabla_{k} K=0$.

Hence we get from (3.3)

$$
\begin{aligned}
G \cong & 2 \nabla_{q} \nabla^{q} D_{j i}\left\{\nabla^{j} \nabla^{i} D_{p}^{p}+\nabla_{p} \nabla^{p} D^{j i}+\frac{2 K}{n(n-1)}\left(g^{j i} D_{p}^{p}-D^{j i}\right)\right\} \\
& -\frac{2 K}{n} D_{j i} \nabla_{q} \nabla^{q} D^{j i} \\
& -\frac{2 K}{n(n-1)}\left(D_{q}^{q} g_{j i}-D_{j i}\right)\left\{\frac{2 K}{n} D^{j i}-\frac{2 K}{n(n-1)}\left(D_{p}^{p} g^{j i}-D^{j i}\right)\right. \\
& \left.-\nabla_{p} \nabla^{p} D^{j i}-\nabla^{j} \nabla^{i} D_{p}^{p}\right\} \\
& +\frac{4 K^{2}}{n^{2}(n-1)} D_{j i} D^{j i}
\end{aligned}
$$




$$
\begin{aligned}
& -\frac{4 K}{n(n-1)} D_{j i}\left(\nabla_{q} \nabla^{j} D^{i q}-\nabla_{q} \nabla^{q} D^{j i}\right) \\
& +\frac{4 K^{2}}{n^{2}(n-1)^{2}}\left\{(n-2) D_{j i} D^{j i}+\left(D_{p}^{p}\right)^{2}\right\} \\
& -\frac{2 K}{n(n-1)} D_{q}^{q} \nabla_{r} \nabla^{r} D_{p}^{p}-\frac{2 K^{2}}{n^{2}(n-1)}\left(D_{p}^{p}\right)^{2} \\
& -\frac{4 K^{2}}{n^{2}(n-1)} D_{j i} D^{j i}
\end{aligned}
$$

since such terms as $D_{j i} \nabla^{j} \nabla^{i} D_{p}^{p}$ are divergences because of (3.1).

We use Ricci's identities and identities such as (3.2) and get

$$
\begin{aligned}
2 \nabla_{q} \nabla^{q} D_{j i} \nabla^{j} \nabla^{i} D_{p}^{p} & \cong-2 \nabla^{j} \nabla_{q} \nabla^{q} D_{j i} \nabla^{i} D_{p}^{p} \\
& \cong-\frac{4 K}{n(n-1)} D_{p}^{p} \nabla_{q} \nabla^{q} D_{r}^{r}
\end{aligned}
$$

by virtue of (3.1). We also get

and finally

$$
D_{j i} \nabla_{p} \nabla^{j} D^{i p} \cong \frac{K}{n(n-1)}\left\{D_{j i} D^{j i}-\left(D_{p}^{p}\right)^{2}\right\}+\frac{K}{n} D_{j i} D^{j i}
$$

$$
\begin{aligned}
G \cong & 2 \nabla_{q} \nabla^{q} D_{j i} \nabla_{p} \nabla^{p} D^{j i} \\
& -\frac{2 K}{n-1}\left(D_{j i} \nabla_{p} \nabla^{p} D^{j i}-\frac{1}{n} D_{p}^{p} \nabla_{q} \nabla^{q} D_{r}^{r}\right) \\
& +\frac{4(n-2)}{n^{2}(n-1)^{2}} K^{2} D_{j i} D^{j i} \\
& -\frac{2(n-3)}{n^{2}(n-1)^{2}} K^{2}\left(D_{p}^{p}\right)^{2} .
\end{aligned}
$$

\section{$\S 5$. Proof of the main theorem.}

If we put

$$
D_{j i}=H_{j i}+\frac{H}{n} g_{j i}, \quad H=D_{p}^{p},
$$

we get $g{ }^{j i} H_{j i}=0$. Let us define $G_{1}, G_{2}$ by

$$
\begin{aligned}
G_{1} & =2\left[\nabla_{q} \nabla^{q} H_{j i} \nabla_{p} \nabla^{p} H^{j i}-\frac{K}{n-1} H_{j i} \nabla_{p} \nabla^{p} H^{j i}+\frac{2(n-2)}{n^{2}(n-1)^{2}} K^{2} H_{j i} H^{j i}\right], \\
G_{2} & =\frac{2}{n}\left[\nabla_{q} \nabla^{q} H \nabla_{p} \nabla^{p} H-\frac{n-4}{n^{2}(n-1)} K^{2} H^{2}\right] .
\end{aligned}
$$

Then we have $G \cong G_{1}+G_{2}$ and

$$
I^{\prime \prime}[g(0)]=\int G_{1} d V+\int G_{2} d V .
$$


Writing $\Delta f=-\nabla_{p} \nabla^{p} f$ we get

$$
\begin{aligned}
\int(\Delta f)^{2} d V & =\int f \nabla_{q} \nabla^{q} \nabla^{p} \nabla_{p} f d V \\
& =\int f \nabla_{q}\left(\nabla^{p} \nabla^{q} \nabla_{p} f-K_{p}^{q} \nabla^{p} f\right) d V \\
& =\int\left(\nabla^{q} \nabla^{p} f\right)\left(\nabla_{q} \nabla_{p} f\right) d V+\frac{K}{n} \int f \Delta f d V .
\end{aligned}
$$

Since we have

$$
\left(\nabla^{q} \nabla^{p} f+\frac{1}{n} g^{q p} \Delta f\right)\left(\nabla_{q} \nabla_{p} f+\frac{1}{n} g_{q p} \Delta f\right)=\left(\nabla^{q} \nabla^{p} f\right)\left(\nabla_{q} \nabla_{p} f\right)-\frac{1}{n}(\Delta f)^{2},
$$

we get

$$
\begin{aligned}
\int(\Delta f)^{2} d V= & \int\left(\nabla^{q} \nabla^{p} f+\frac{1}{n} g^{q p} \Delta f\right)\left(\nabla_{q} \nabla_{p} f+\frac{1}{n} g_{q p} \Delta f\right) d V \\
& +\frac{1}{n} \int(\Delta f)^{2} d V+\frac{K}{n} \int f \Delta f d V,
\end{aligned}
$$

hence

$$
\int(\Delta f)^{2} d V \geqq \frac{K}{n-1} \int \nabla_{p} f \nabla^{p} f d V .
$$

It is well-known [3] that, if $\lambda_{1}$ is the first positive eigenvalue of the equation $\Delta \rho=\lambda \rho$, and if $f$ satisfies $\int f d V=0$, then

$$
\int \nabla_{p} f \nabla^{p} f d V \geqq \lambda_{1} \int f^{2} d V
$$

Moreover,

$$
\lambda_{1}=\frac{1}{n-1} K
$$

for a space of positive constant curvature [5], [7]. Hence we have

$$
\int(\Delta f)^{2} d V \geqq \frac{1}{(n-1)^{2}} K^{2} \int f^{2} d V
$$

in our case.

If $H$ does not vanish identically, we have

$$
\int(\Delta H)^{2} d V-\frac{n-4}{n^{2}(n-1)} K^{2} \int H^{2} d V \geqq\left[\frac{1}{(n-1)^{2}}-\frac{n-4}{n^{2}(n-1)}\right] K^{2} \int H^{2} d V>0,
$$

hence $\int G_{2} d V>0$. Since we have $\int G_{1} d V \geqq 0$ always, we have proved $\int\left(G_{1}+G_{2}\right) d V>0$ in this case.

Let us now assume $H \equiv 0$. If moreover $H_{j i}$ also vanishes everywhere on $M$, we have $D_{j i} \equiv 0$. We need not consider this case. If $H_{j i}$ does not 
identically vanish, we have $\int G_{1} d V>0$, hence $\int\left(G_{1}+G_{2}\right) d V>0$.

Thus we have proved the main theorem.

\section{References}

[1] M. Berger, Quelques formules des variation pour une structure riemannienne, Ann. sci. École Norm. Sup., $4^{\text {e }}$ série, 3 (1970), 285-294.

[2] M. Berger and D. Ebin, Some decompositions of the space of symmetric tensors on a Riemannian manifold, J. Differential Geometry, 3 (1969), 379-392.

[3] M. Berger, P. Gauduchon and E. Mazet, Le spectre d'une variété riemannienne, Springer, 1971.

[4] D. Ebin, The manifolds of Riemannian metrics, Proceedings of 1968 AMS Summer Institute on Global Analysis.

[5] A. Lichnerowicz, Géométrie des groupes de transformations, Dunod, 1958.

[6] Y. Mutō, On Einstein metrics, J. Differential Geometry, to appear.

[7] M. Obata, Certain conditions for a Riemannian manifold to be isometric with a sphere, J. Math. Soc. Japan, 14 (1962), 333-340.

\section{Yosio Mutō}

Department of Applied Mathematics

Faculty of Engineering

Yokohama National University

Ooka-2-chome, Minami-ku

Yokohama, Japan 